\title{
Quantitative Assessment the Relationship between p21 rs1059234 Polymorphism and Cancer Risk
}

\author{
Yong-Sheng Huang, Qian-Qian Fan, Chuang Li, Meng Nie, Hong-Yang Quan, \\ Lin Wang*
}

\begin{abstract}
p21 is a cyclin-dependent kinase inhibitor, which can arrest cell proliferation and serve as a tumor suppressor. Though many studies were published to assess the relationship between p21 rs1059234 polymorphism and various cancer risks, there was no definite conclusion on this association. To derive a more precise quantitative assessment of the relationship, a large scale meta-analysis of 5,963 cases and 8,405 controls from 16 eligible published case-control studies was performed. Our analysis suggested that rs1059234 was not associated with

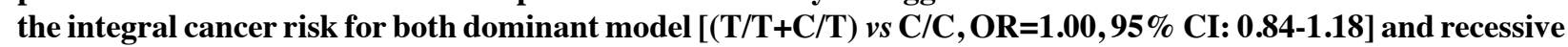
model [T/T vs $(\mathrm{C} / \mathrm{C}+\mathrm{C} / \mathrm{T}), \mathrm{OR}=1.03,95 \% \mathrm{CI}$ : 0.93-1.15)]. However, further stratified analysis showed rs1059234 was greatly associated with the risk of squamous cell carcinoma of head and neck (SCCHN). Thus, larger scale primary studies are still required to further evaluate the interaction of p21 rs1059234 polymorphism and cancer risk in specific cancer subtypes.
\end{abstract}

Keywords: p21 - cancer risk - polymorphism - meta-analysis

Asian Pac J Cancer Prev, 16 (10), 4435-4438

\section{Introduction}

It has been suggested that environmental and genetic factors may affect the individual's susceptibility to cancer(Derynck et al., 2001). An important gene identified as cancer susceptibility one is p21 (also known as CDKN1A), a member of the Cip/Kip family of cyclindependent kinase (CDK) inhibitors. Expression of p21 is up-regulated by wildtype p53 in response to DNA damage to induce cell cycle arrest at the G1 checkpoint (Xiong et al., 1993; Sherr, 1996). p21 can exerts tumorsuppressive effects by inhibiting PCNA-dependent DNA replication and mismatch repair(Li et al., 1994; Waga et al., 1997). Somatic mutations in the p21 gene are rare in human malignancies(Roninson, 2002). However, reduced p21 expression in tumors has been associated with poor prognosis in humans (Jiang et al., 1997; Wakasugi et al., 1997). Therefore, genetic polymorphisms in p21 may modulate its expression and thereby affect carcinogenesis.

p21 polymorphism rs1059234 (C70T) locates within the 3' untranslated region of p21 gene, causes a single C-to-T substitution $20 \mathrm{nt}$ downstream of the stop codon at exon 3 (http://egp.gs.washington.edu). This polymorphic variant identified was thought to alter p21 function and maybe functionally associated with cancer susceptibility.

Analysis of case-control studies is the most prevalent method of investigating the association between a disease and a specific gene polymorphism. Thus far, a number of studies have reported the role of p21 rs1059234 polymorphism in cancer risk (Li et al., 2005; Lei et al., 2010), but the results remain conflicting (Sivonova et al., 2013; Yin et al., 2015), partially because of the relatively small sample size in each of the published studies. Therefore, here we performed a large scale meta-analysis of all the published studies to derive a more precise quantitative assessment of the association between p21 rs1059234 polymorphism and the cancer risk.

\section{Materials and Methods}

Selection of studies

All of the case-control studies were identified by a computerized literature search of the PubMed, Web of Science, EBSCO, and CGEMS database (prior to March 2015) using the following words and terms: "p21", "CDKN1A", "polymorphism", and "cancer". References of the retrieved publications were also screened. Studies had to be based on an unrelated case-control design, so pedigree data were excluded. The following basic data were collected from the studies: first authors, journals, year of publications, cancer subtypes and ethnicity of the population.

\section{Statistical analysis}

For each study, the OR was first calculated to assess the association between the polymorphisms and the disease 
in table 1. In meta-analysis, we examined the association between p21 rs1059234 polymorphism and the risk of cancer using recessive $[\mathrm{T} / \mathrm{T}$ v $(\mathrm{C} / \mathrm{C}+\mathrm{C} / \mathrm{T})]$ and dominant $[(\mathrm{T} / \mathrm{T}+\mathrm{C} / \mathrm{T}) v s \mathrm{C} / \mathrm{C}]$ genetic models. There are three widely used methods of meta-analysis for dichotomous outcomes: two fixed effects methods (Mantel-Haenszel's method and Peto's method), which assume that studies are sampled from populations with the same effect size, making an adjustment to the study weights according to the in-study variance; and one random effects method (DerSimonian and Laird's method), which assumes that studies are taken from populations with varying effect sizes, calculating the study weights both from the in-study and between-study variance, considering the extent of variation, or heterogeneity. In our study, both Mantel-Haenszel's fixed effects method and DerSimonian and Laird's random effects method were used in Stata 10.0 software. A chi-square-based Q-statistic test was performed to evaluate the between-study heterogeneity of the studies. If $P<0.10$, the between- study heterogeneity was considered to be significant, we chose the randomeffects model to calculate the OR. Otherwise, when $P \geq 0.10$, the between-study heterogeneity was not significant, then the fixed-effects model was suitable. In the absence of between-study heterogeneity, the two methods yield similar results. In order to make a clear comparison, we present the OR of both the random-effects model and fixed-effects model for every meta-analysis. A pooled OR obtained by meta-analysis was used to give a more reasonable evaluation of the association. A Z test was performed to determine the significance of the pooled $\mathrm{OR}(P \leq 0.05$ suggests a significant $\mathrm{OR})$. Funnel plots were used to access publication bias by the method of Egger's regression test. A $\mathrm{T}$ test was performed to determine the significance of the asymmetry. An asymmetric plot suggested possible publication bias $(P \geq 0.05$ suggests no bias). Hardy-Weinberg equilibrium was tested by the
Chisquare test based on a program (http://www.ihg.gsf. de/cgi-bin/hw/hwa1.pl). Analyses were performed by Stata10.0 software.

\section{Results}

\section{Study characteristics}

There are 16 studies (5,963 cases and 8,405 controls) analyzing the relation of p21 rs 1059234 polymorphism and the risk of cancer. Each subpopulation in these articles was treated as a separate study in our meta-analysis. All the studies were published from Year 2005 to 2015. Populations were divided into different cancer subtypes and ethnic categories. Table 1 shows the details of the cases and controls in the included studies, together with the ORs we calculated to make a primary evaluation. Table 2 is the summary of the meta-analysis of case-control studies examining the association between p21 rs 1059234 polymorphism and cancer risk, with the comparison between different cancer subtypes and different ethnicities.

\section{Main results}

For each study we investigated the association between p21 rs1059234 polymorphism and cancer risk, assuming different inheritance models of the C70T allele. Overall, when all the eligible studies were pooled into the meta-analysis, no associations between p21 rs1059234 polymorphism and cancer susceptibility were observed in all genetic models. No significant associations were found for T/T vs $\mathrm{C} / \mathrm{C}(\mathrm{OR}=1.02 ; 95 \% \mathrm{CI}$ : 0.83-1.26; $P=0.010$ for heterogeneity), $\mathrm{C} / \mathrm{T}$ vs $\mathrm{C} / \mathrm{C}(\mathrm{OR}=0.98$; $95 \% \mathrm{CI}$ : 0.82-1.16; $P=0.000$ for heterogeneity), $\mathrm{T} / \mathrm{T}+\mathrm{C} / \mathrm{T}$ vs $\mathrm{C} / \mathrm{C}(\mathrm{OR}=1.00$; 95\% CI: $0.84-1.18 ; P=0.000$ for heterogeneity) and T/T vs $\mathrm{C} / \mathrm{T}+\mathrm{T} / \mathrm{T}(\mathrm{OR}=1.03$; $95 \% \mathrm{CI}$ : 0.93-1.15; $P=0.135$ for heterogeneity)(Table 2). However, subgroup analyses by cancer type showed rs 1059234 polymorphism might associate with the risk of SCCHN for $\mathrm{T} / \mathrm{T}+\mathrm{C} / \mathrm{T}$ vs $\mathrm{C} / \mathrm{C}$

Table 1. Characteristics of Studies Included in the Meta-Analysis

\begin{tabular}{|c|c|c|c|c|c|c|c|c|c|c|}
\hline \multirow[b]{3}{*}{ Author } & \multirow[b]{3}{*}{ Cancer subtype } & \multicolumn{8}{|c|}{ enotype distribution of p21 rs 1059234 polymorphism } & \multirow{3}{*}{$\begin{array}{l}\text { HWE } \\
\text { (control } \\
P \text { value) }\end{array}$} \\
\hline & & \multirow[b]{2}{*}{ Population } & \multicolumn{2}{|r|}{ Case } & \multicolumn{3}{|c|}{ Control } & \multicolumn{2}{|c|}{ OR $(95 \% \mathrm{CI})$} & \\
\hline & & & $\overline{\mathrm{C} / \mathrm{C}}$ & $\overline{\mathrm{C} / \mathrm{T} \mathrm{T} / \mathrm{T}}$ & $\overline{\mathrm{C} / \mathrm{C}}$ & $\mathrm{C} / \mathrm{T}$ & $\overline{\mathrm{T} / \mathrm{T}}$ & $\begin{array}{c}\mathrm{T} / \mathrm{T} v s \\
(\mathrm{C} / \mathrm{C}+\mathrm{C} / \mathrm{T})\end{array}$ & $\begin{array}{c}(\mathrm{T} / \mathrm{T}+\mathrm{C} / \mathrm{T}) \\
v s \mathrm{C} / \mathrm{C}\end{array}$ & \\
\hline Li et al., 2005 & SCCHN & Caucasian & 596 & 110 & 1080 & 136 & 6 & $1.72(0.55-5.36)$ & $1.48(1.14-1.93)$ & 0.445 \\
\hline Wu et al., 2006 & bladder cancer & Caucasian & 513 & 86 & 506 & 82 & 3 & $0.98(0.20-4.88)$ & $1.03(0.75-1.42)$ & 0.8692 \\
\hline Ma et al., 2006 & Breast cancer & Asian & 87 & 21170 & 129 & 253 & 85 & $1.06(0.74-1.50)$ & $1.23(0.90-1.69)$ & 0.044 \\
\hline Guo et al., 2006 & ESCC & Asian & 94 & 15451 & 166 & 221 & 50 & $1.59(1.04-2.43)$ & $1.34(0.98-1.82)$ & 0.0655 \\
\hline Guo et al., 2006 & gastric cancer & Asian & 95 & 12150 & 166 & 221 & 50 & $1.79(1.17-2.74)$ & $1.10(0.80-1.51)$ & 0.0655 \\
\hline Driver et al., 2008 & prostate cancer & Caucasian & 167 & 18 & 181 & 39 & 1 & $1.19(0.07-19.14)$ & $0.51(0.29-0.92)$ & 0.4711 \\
\hline Polakova et al., 2009 & Colorectal Cancer & Caucasian & 534 & 69 & 520 & 89 & 1 & $4.04(0.45-36.25)$ & $0.79(0.57-1.10)$ & 0.1603 \\
\hline Lei et al., 2010) & $\mathrm{SCCHN}$ & Caucasian & 93 & 25 & 1009 & 139 & 14 & $1.39(0.31-6.19)$ & $1.91(1.21-3.04)$ & 0.0004 \\
\hline Liu et al., 2010 & Colorectal Cancer & Asian & 100 & 19776 & 223 & 438 & 177 & 0.96(0.71-1.29) & $0.99(0.75-1.30)$ & 0.1603 \\
\hline Taghavi et al., 2010 & ESCC & Caucasian & 99 & 27 & 82 & 18 & 0 & Excluded & $0.61(0.45-0.83)$ & 0.3227 \\
\hline Wang et al., 2012 & cervical cancer & Asian & 131 & 160102 & 102 & 221 & 111 & $1.02(0.75-1.39)$ & $1.05(0.80-1.38)$ & 0.6942 \\
\hline Liu et al., 2013 & hepatocellular cancer & Asian & 134 & 224118 & 153 & 255 & 118 & $1.14(0.85-1.52)$ & $2.15(1.23-3.76)$ & 0.5493 \\
\hline Carvalho et al., 2013 & retinoblastoma & Mixed (Braz) & 90 & 49 & 95 & 23 & 2 & $0.85(0.12-6.12)$ & $0.88(0.60-1.13)$ & 0.6599 \\
\hline Sivonova et al., 2013 & prostate cancer & Caucasian & 104 & 14 & 108 & 22 & 0 & Excluded & $0.78(0.58-1.05)$ & 0.2919 \\
\hline Zheng et al., 2014 & ESCC & Asian & 172 & 321107 & 170 & 340 & 141 & $0.79(0.59-1.04)$ & $0.56(0.39-0.81)$ & 0.2342 \\
\hline Shao et al., 2014 & gastric cancer & Asian & 99 & 15856 & 154 & 301 & 126 & $0.79(0.55-1.12)$ & $1.24(0.64-2.41)$ & 0.3527 \\
\hline Yin et al., 2015 & Endometrial Cancer & Asian & 88 & 11065 & 69 & 165 & 81 & $0.95(0.65-1.38)$ & $0.66(0.32-1.36)$ & 0.3831 \\
\hline
\end{tabular}

*OR: Odds ratio, CI: confidence interval, HWE: Hardy-Weinberg equilibrium, SCCHN: Squamous cell carcinoma of the head and neck, ESCC: esophageal squamous cell carcinoma 
Table 2. Summary of the Meta-analysis of Case-control Studies Examining the Association between p21 rs1059234 Polymorphism and Cancer Risk

\begin{tabular}{lcccc}
\hline $\mathrm{OR}(95 \% \mathrm{CI})$ & $\mathrm{T} / \mathrm{T}$ vs $\mathrm{C} / \mathrm{C}$ & $\mathrm{C} / \mathrm{T} v s \mathrm{C} / \mathrm{C}$ & $\mathrm{T} / \mathrm{T} v s(\mathrm{C} / \mathrm{C}+\mathrm{C} / \mathrm{T})$ & $(\mathrm{T} / \mathrm{T}+\mathrm{C} / \mathrm{T}) v s \mathrm{~T} / \mathrm{T}$ \\
\hline Studies & & & & \\
All of studies & $1.02(0.83-1.26)$ & $0.98(0.82-1.16)$ & $1.03(0.93-1.15)$ & $1.00(0.84-1.18)$ \\
All of SCCHN studies & $1.72(0.70-4.23)$ & $1.56(1.23-1.98)$ & $1.60(0.65-3.92)$ & $1.57(1.25-1.98)$ \\
All of ESCCstudies & $1.14(0.48-2.70)$ & $1.06(0.87-1.28)$ & $1.10(0.55-2.19)$ & $1.05(0.87-1.27)$ \\
All of colorectal Cancer studies & $1.00(0.71-1.43)$ & $0.89(0.71-1.11)$ & $0.99(0.74-1.33)$ & $0.90(0.73-1.11)$ \\
All of gastric cancerstudies & $1.09(0.44-2.71)$ & $0.88(0.70-1.11)$ & $1.17(0.53-2.63)$ & $0.92(0.74-1.14)$ \\
All of Caucasian studies & $1.67(0.82-3.37)$ & $1.02(0.74-1.41)$ & $1.61(0.80-3.25)$ & $1.03(0.75-1.42)$ \\
All of Asian studies & $0.98(0.78-1.25)$ & $0.89(0.75-1.07)$ & $1.05(0.89-1.24)$ & $0.92(0.77-1.10)$ \\
\hline
\end{tabular}

A)

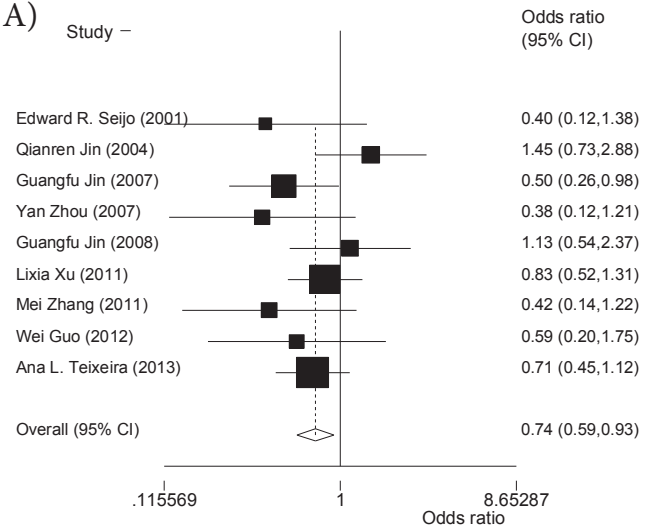

B)

\% Weight

3.9
7.8
14.6
6.2
7.3
22.9
6.5
5.5
25.4

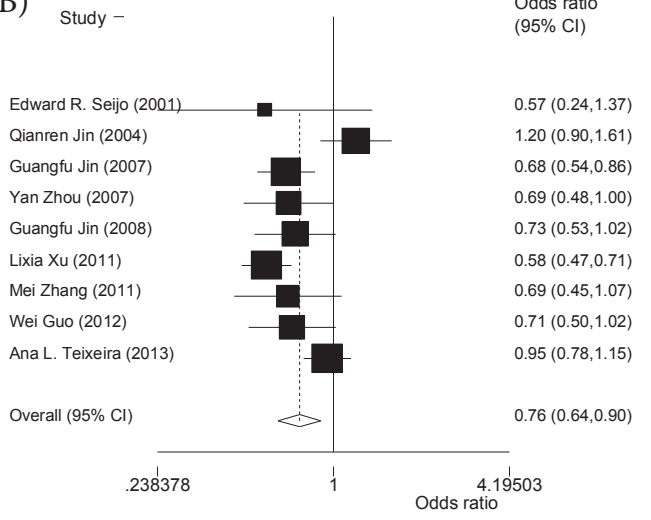

Figure 1. Forest Plot of Cancer Risk Associated with the p21 rs1059234 Polymorphism. The squares and horizontal lines correspond to the study-specific OR and $95 \% \mathrm{CI}$. The area of the squares reflects the weight (inverse of the variance). The diamond represents the summary OR and $95 \% \mathrm{CI}$. A) recessive genetic model [T/T $v s(\mathrm{~T} / \mathrm{T}+\mathrm{C} / \mathrm{T})] \mathbf{B})$ dominant genetic models [(T/ $\mathrm{T}+\mathrm{C} / \mathrm{T})$ vs $\mathrm{C} / \mathrm{C}]$

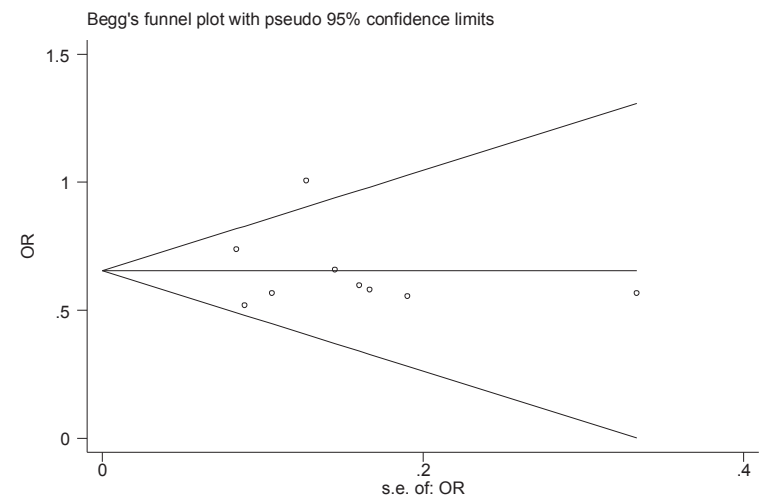

Figure 2. Begg's Funnel Plot of the Egger's Test for Publication Bias in Comparison of rs1059234 Polymorphism C70T Allele T vs allele C

(OR=1.57; 95\% CI: $1.25-1.98 ; P=0.342$ for heterogeneity) and $\mathrm{T} / \mathrm{T}$ vs $\mathrm{C} / \mathrm{T}+\mathrm{C} / \mathrm{C}(\mathrm{OR}=1.60$; 95\% CI: 0.65-3.92; $P=0.823$ for heterogeneity) (Table 2).

\section{Sensitivity analyses and publication bias}

The results suggested the influences of the individual data set to the pooled ORs are all not significant. Funnel plots and Egger's test were performed to assess publication bias (Figure 2). The data suggested that there is no publication bias for the comparison of rs1059234 polymorphism C70T allele $\mathrm{T} v$ s allele $\mathrm{C}(\mathrm{t}=1.03, P=0.318)$.

\section{Discussion}

Cell cycle control is crucial for normal cell growth and differentiation and is regulated by cyclin-dependent kinases (CDKs). p21 is one of the universal inhibitors of cyclin-dependent kinases (CDK2, CDK3, CDK4, and CDK6)(Gartel and Tyner, 2002). It was initially discovered as a p53-target gene, but also has been suggested to play a role as a tumor suppressor in other cellular pathways including TGF- $\beta$ and Wnt (Englert et al., 1997; Suzuki et al., 2012). Given the functional importance of p21 in carcinogenesis, genetic alteration of p21 could be associated with cancer risk.

So far, the functional role of the $\mathrm{p} 21 \mathrm{rs} 1059234$ variant has not yet to be well interpreted, several published clinic studies reported this variant was at increased risk of developing various cancer(Li et al., 2005; Lei et al., 2010; Liu et al., 2013). However, a number of published clinic studies reported this variant was not involved in the risk of cancer (Wu et al., 2006; Zheng et al., 2014; Yin et al., 2015). These conflicting studies based their conclusions on a small number of samples, so a metaanalysis of all available studies will help to establish a more convincing result. From our meta-analysis, p21 rs1059234 polymorphism in the combined population did not associate with cancer risk. There is no publication bias among the total studies. However, in the stratified analysis by ethnicity and subtype of cancer, significant association between p21 rs 1059234 polymorphism and the risk of SCCHN was detected.

In conclusion, the research of the relationship of p21 rs 1059234 polymorphism and cancer is very popular but remain conflicting at present. Our meta-analysis suggested that under recessive, dominant and other genetic models, 


\section{Yongsheng Huang et al}

the p21 rs1059234 polymorphism did not associated with integral cancer risk. However, the studies included in the subgroups analysis are still limited and the results are sensitive to study selection. Since p21 also has a dual role can assume both pro- or anti-apoptotic functions in response to anti-tumor agents, depending on the cell type and context (Liu et al., 2003; Gartel, 2005). More comparative studies are needed to evaluate interactions of p21 rs1059234 polymorphism and cancer risk in specific cancer subtypes, especially in SCCHN.

\section{References}

Carvalho IN, Reis AH, Cabello PH, et al (2013). Polymorphisms of CDKN1Agene and risk of retinoblastoma. Carcinogenesis, 34, 2774-7.

Derynck R, Akhurst RJ, Balmain A (2001). TGF-beta signaling in tumor suppression and cancer progression. Nat Genet, 29, 117-29.

Driver KE, Song H, Lesueur F, et al (2008). Association of single-nucleotide polymorphisms in the cell cycle genes with breast cancer in the British population. Carcinogenesis, 29, 333-41.

Englert C, Maheswaran S, Garvin AJ, et al (1997). Induction of p21 by the Wilms' tumor suppressor gene WT1. Cancer Res, 57, 1429-34.

Gartel AL (2005). The conflicting roles of the cdk inhibitor p21(CIP1/WAF1) in apoptosis. Leuk Res, 29, 1237-8.

Gartel AL, Tyner AL (2002). The role of the cyclin-dependent kinase inhibitor p21 in apoptosis. Mol Cancer Ther, 1, 639-49.

Guo W, Cui YJ, Fang SM, et al (2006). [Association of polymorphisms of p21cip1 and p27kip1 genes with susceptibilities of esophageal squamous cell carcinoma and gastric cardiac adenocarcinoma]. Ai Zheng, 25, 194-9.

Jiang M, Shao ZM, Wu J, et al (1997). p21/waf1/cip1 and mdm-2 expression in breast carcinoma patients as related to prognosis. Int J Cancer, 74, 529-34.

Lei D, Sturgis EM, Liu Z, et al (2010). Genetic polymorphisms of p21 and risk of second primary malignancy in patients with index squamous cell carcinoma of the head and neck. Carcinogenesis, 31, 222-7.

Li G, Liu Z, Sturgis EM, et al (2005). Genetic polymorphisms of p21 are associated with risk of squamous cell carcinoma of the head and neck. Carcinogenesis, 26, 1596-602.

Li R, Waga S, Hannon GJ, et al (1994). Differential effects by the p21 CDK inhibitor on PCNA-dependent DNA replication and repair. Nature, $\mathbf{3 7 1}, 534-7$.

Liu B, Zhang Y, Jin M, et al (2010). Association of selected polymorphisms of CCND1, p21, and caspase 8 with colorectal cancer risk. Mol Carcinog, 49, 75-84.

Liu F, Wei YG, Luo LM, et al (2013). Genetic variants of p21 and p27 and hepatocellular cancer risk in a Chinese Han population: a case-control study. Int J Cancer, 132, 2056-64.

Liu S, Bishop WR, Liu M (2003). Differential effects of cell cycle regulatory protein $\mathrm{p} 21$ (WAF1/Cip1) on apoptosis and sensitivity to cancer chemotherapy. Drug Resist Updat, 6, 183-95.

$\mathrm{Ma} \mathrm{H}$, Jin G, Hu Z, et al (2006). Variant genotypes of CDKN1 A and CDKN1B are associated with an increased risk of breast cancer in Chinese women. Int J Cancer, 119, 2173-8.

Polakova V, Pardini B, Naccarati A, et al (2009). Genotype and haplotype analysis of cell cycle genes in sporadic colorectal cancer in the Czech Republic. Hum Mutat, 30, 661-8.

Roninson IB (2002). Oncogenic functions of tumour suppressor p21(Waf1/Cip1/Sdi1): association with cell senescence and tumour-promoting activities of stromal fibroblasts. Cancer Lett, 179, 1-14.

Shao A, Zheng L, Chen S, et al (2014). p21, p53, TP53BP1 and p73 polymorphisms and the risk of gastric cardia adenocarcinoma in a Chinese population. Biomarkers, 1-7.

Sherr CJ (1996). Cancer cell cycles. Science, 274, 1672-7.

Sivonova MK, Vilckova M, Jurecekova J, et al (2013). The role of p21 3'UTR gene polymorphism in the risk of prostate cancer: a pilot study. Mol Med Rep, 7, 986-90.

Suzuki H, Ito R, Ikeda K, et al (2012). TATA-binding protein (TBP)-like protein is required for p53-dependent transcriptional activation of upstream promoter of $\mathrm{p} 21 \mathrm{Waf} 1 /$ Cipl gene. J Biol Chem, 287, 19792-803.

Taghavi N, Biramijamal F, Abbaszadegan MR, et al (2010). P21(waf1/cip1) gene polymorphisms and possible interaction with cigarette smoking in esophageal squamous cell carcinoma in northeastern Iran: a preliminary study. Arch Iran Med, 13, 235-42.

Waga S, Li R, Stillman B (1997).p53-induced p21 controls DNA replication. Leukemia, 11, 321-3.

Wakasugi E, Kobayashi T, Tamaki Y, et al (1997). p21(Waf1/ Cip1) and $\mathrm{p} 53$ protein expression in breast cancer. Am J Clin Pathol, 107, 684-91.

Wang N, Wang S, Zhang Q, et al (2012). Association of p21 SNPs and risk of cervical cancer among Chinese women. BMC Cancer, 12, 589.

Wu X, Gu J, Grossman HB, et al (2006). Bladder cancer predisposition: a multigenic approach to DNA-repair and cell-cycle-control genes. Am J Hum Genet, 78, 464-79.

Xiong Y, Hannon GJ, Zhang H, et al (1993). p21 is a universal inhibitor of cyclin kinases. Nature, 366, 701-4.

Yin D, Jiang Y, Zhang S, et al (2015). No association between p21 Gene rs1059234 polymorphisms and risk of endometrial cancer among han women in northeast China. Cell Biochem Biophys, 71, 167-71.

Zheng L, Tang W, Shi Y, et al (2014). p21 rs3176352 G>C and p73 rs1801173 C > T polymorphisms are associated with an increased risk of esophageal cancer in a Chinese population. PLoS One, 9, 96958. 